\title{
THE PROPAGATION OF WAVES IN ORTHOTROPIC MEDIA*
}

\author{
BY \\ G. F. CARRIER \\ Harvard University
}

1. Introduction. The present article is an extension of a previous paper dealing with the elasticity problems of orthotropic media. ${ }^{1}$ Here, the displacement potentials which define the dynamic phenomena in such media are discussed.

2. The dynamic problem. Hooke's law for an orthotropic medium may be written in the form

$$
\begin{aligned}
\sigma_{x} & =b_{11} e_{x}+b_{12} e_{y}+b_{13} e_{z}, \cdots, \\
\tau_{x y} & =b_{66} \gamma_{x y}, \cdots,
\end{aligned}
$$

wherein we use the conventional notation for the stresses and the strains. If we limit ourselves to a consideration of those materials which are isotropic in the $y, z$ plane $^{2}$ and for which

$$
b_{66}=b_{65}=\left(b_{11} b_{22}-b_{12}^{2}\right) /\left(b_{11}+b_{22}+{ }_{2} b_{12}\right),
$$

the number of independent elastic constants is reduced to four and the dynamic elasticity problem may be easily treated. ${ }^{3}$ We utilize the familiar equilibrium equations

$$
\frac{\partial \sigma_{x}}{\partial x}+\frac{\partial \tau_{x y}}{\partial y}+\frac{\partial \tau_{x z}}{\partial z}+\rho X-\rho \frac{\partial^{2} u}{\partial t^{2}}=0, \cdots,
$$

and define the displacements in terms of potentials as

$$
\begin{aligned}
e_{x} & =\frac{\partial u}{\partial x}=\frac{\partial^{2} \phi_{1}}{\partial x^{2}}, \ldots, \\
\gamma_{x y} & =\frac{\partial u}{\partial y}+\frac{\partial v}{\partial x}=\frac{\partial^{2}}{\partial x \partial y}\left(\phi_{1}+\phi_{2}\right), \cdots .
\end{aligned}
$$

When Eqs. (1), (2), and (3) are combined, we obtain

$$
\begin{gathered}
\frac{\partial}{\partial x}\left\{\left[a \frac{\partial^{2}}{\partial x^{2}}+\alpha\left(\frac{\partial^{2}}{\partial y^{2}}+\frac{\partial^{2}}{\partial z^{2}}\right)-\frac{\partial^{2}}{\partial t^{2}}\right] \phi_{1}+\beta\left(\frac{\partial^{2} \phi_{2}}{\partial y^{2}}+\frac{\partial^{2} \phi_{3}}{\partial z^{2}}\right)\right\}+X=0, \\
\frac{\partial}{\partial y}\left\{\beta \frac{\partial^{2} \phi_{1}}{\partial x^{2}}+\left(\alpha \frac{\partial^{2}}{\partial x^{2}}+b \frac{\partial^{2}}{\partial y^{2}}+\gamma \frac{\partial^{2}}{\partial z^{2}}-\frac{\partial^{2}}{\partial t^{2}}\right) \phi_{2}+\delta \frac{\partial^{2} \phi_{3}}{\partial z^{2}}\right\}+Y=0, \\
\frac{\partial}{\partial z}\left\{\beta \frac{\partial^{2} \phi_{1}}{\partial x^{2}}+\delta \frac{\partial^{2} \phi_{2}}{\partial y^{2}}+\left(\alpha \frac{\partial^{2}}{\partial x^{2}}+\gamma \frac{\partial^{2}}{\partial y^{2}}+b \frac{\partial^{2}}{\partial z^{2}}-\frac{\partial^{2}}{\partial t^{2}}\right) \phi_{3}\right\}+Z=0,
\end{gathered}
$$

* Received August 18, 1945.

1 G. F. Carrier, The thermal stress and body force problems of the infinite orthotropic solid, Quart. Appl. Math. 2, 31-36 (1944).

2 The isotropy implies that $b_{22}=b_{33}, b_{12}=b_{13}, b_{14}=\left(b_{22}-b_{23}\right) / 2$.

${ }^{3}$ These conditions are imposed in order that the roots of Eq. (8) will appear in a useful form. They include isotropic media as a special case. 
where $a, b, \alpha, \gamma, \beta^{2}$, and $\delta$, are given respectively by $b_{11} / \rho, b_{22} / \rho, b_{66} / \rho,\left(b_{22}-b_{33}\right) / 2 \rho$, $(a-\alpha)(b-\alpha)$, and $b-\gamma$.

If we now consider the homogeneous equations (i.e., vanishing body forces) and require the $\phi_{i}$ to vanish at infinity, ${ }^{4}$ we may begin the integration by removing the leftmost derivative of each equation, multiplying each term in the remaining forms by $\exp [-i(x \xi+y \eta+z \zeta)]$, and integrating the equations so obtained over the infinite region. We find ${ }^{5}$

$$
\left[a \xi^{2}+\alpha\left(\eta^{2}+\zeta^{2}\right)+\frac{\partial^{2}}{\partial t^{2}}\right] \psi_{1}+\beta \eta^{2} \psi_{2}+\beta \zeta^{2} \psi_{3}=0
$$

and two similar equations. Here

$$
\left.\psi_{j}=\iiint_{-\infty}^{\infty} \phi_{j}(x, y, z, t) \exp [-i(x \xi+y \eta+z\})\right] d x d y d z .
$$

We now have three ordinary linear differential equations (in $t$ ) the solutions to which are of the form

$$
\psi_{j}=A_{j}(\xi, \eta, \zeta) \cos \omega t .
$$

In order that these solutions be non-trivial, the determinant of coefficients of Eqs.(5) wherein $\partial^{2} / \partial t^{2}$ has been replaced by $-\omega^{2}$ must vanish, that is

$$
\left|\begin{array}{ccc}
a \xi^{2}+\alpha\left(\eta^{2}+\zeta^{2}\right)-\omega^{2}, & \beta \eta^{2}, & \beta \zeta^{2} \\
\beta \xi^{2}, & \alpha \xi^{2}+b \eta^{2}+\gamma \zeta^{2}-\omega^{2}, & \delta \zeta^{2} \\
\beta \xi^{2}, & \delta \eta^{2}, & \alpha \xi^{2}+\gamma \eta^{2}+b \zeta^{2}-\omega^{2}
\end{array}\right|=0 .
$$

The three roots of this equation are easily shown to be

$$
\begin{aligned}
& \omega_{1}^{2}=a \xi^{2}+b\left(\eta^{2}+\zeta^{2}\right), \\
& \omega_{2}^{2}=\alpha \xi^{2}+\gamma\left(\eta^{2}+\zeta^{2}\right), \\
& \omega_{3}^{2}=\alpha\left(\xi^{2}+\eta^{2}+\zeta^{2}\right) .
\end{aligned}
$$

Corresponding to these roots, the $A_{k j}$ must respectively obey the relations ${ }^{6}$

$$
\begin{aligned}
& A_{11}: A_{12}: A_{13}=a-\alpha: \beta: \beta, \\
& A_{21}: A_{22}: A_{23}=0: \beta \zeta^{2}:-\beta \eta^{2}, \\
& A_{31}: A_{32}: A_{33}=(b-\alpha)\left(\eta^{2}+\zeta^{2}\right):-\beta \xi^{2}:-\beta \xi^{2} .
\end{aligned}
$$

Because of Eq. (6) it is evident that

$$
\begin{aligned}
\phi_{j} & =\frac{1}{8 \pi^{3}} \iiint_{-\infty}^{\infty} \psi_{j}(\xi, \eta, \zeta, t) \exp [i(x \xi+y \eta+z \zeta)] d \xi d \eta d \zeta \\
& =\sum_{k=1,2,3} \frac{1}{8 \pi^{3}} \iiint_{-\infty}^{\infty} A_{j k}(\xi, \eta, \zeta) \cos \omega_{k} t \exp [i(x \xi+y \eta+z \zeta)] d \xi d \eta d \zeta .
\end{aligned}
$$

4 This condition may be justified by noting that functions describing a time dependent phenomenon which originated at $t_{0}$ in a defined region of the medium must vanish at infinity for all time $t$.

s The procedure thus far has been identically that of the reference in footnote 1 .

- Here, $A_{k j}$ is the $A_{j}$ of Eq. (7) corresponding to $\omega_{k}$. 
The solutions of interest are those for which the $A_{k j}$ are explicit functions of $\omega_{k}$ only. We consider first the solution associated with $\omega_{1}$ and find it convenient to write $A_{11}$ as

$$
A_{11}\left(\omega_{1}\right)=(a-\alpha) \iiint_{-\infty}^{\infty} \frac{P_{11}\left(r_{1}\right)}{r_{1}} \exp \left[i \mathrm{r}_{1} \cdot \omega_{1}\right] d x d y d z .
$$

Here, $\omega_{1}$ is defined as $\omega_{1}=\mathbf{i} a^{1 / 2} \xi+\mathrm{j} b^{1 / 2} \eta+\mathrm{k} b^{1 / 2} \zeta$, and $\mathbf{r}_{1}=\mathbf{i} a^{-1 / 2} x+\mathrm{j} b^{-1 / 2} y+\mathrm{k} b^{-1 / 2} z ; B_{11}$ is an arbitrary function of $r_{1}=\left|\mathbf{r}_{1}\right|$.

If $\eta$ is the angle between $\mathbf{r}_{1}$ and $\omega_{1}$, and $\nu$ is the polar angle about $\omega_{1}$, the value of $A_{11}$ becomes

$$
\begin{aligned}
A_{11} & =(a-\alpha) a^{1 / 2} b \iiint_{-\infty}^{\infty} B_{11}\left(r_{1}\right) \exp \left[i r_{1} \omega_{1} \cos \mu\right] r_{1} \sin \mu d \mu d \nu d r_{1} \\
& =4 \pi(a-\alpha) a^{1 / 2} b \omega_{1}^{-1} \int_{0}^{\infty} B_{11}\left(r_{1}\right) \sin r_{1} \omega_{1} d r_{1} \\
& =4 \pi(a-\alpha) a^{1 / 2} b \omega_{1}^{-1} C_{11}\left(\omega_{1}\right) .
\end{aligned}
$$

Using the same notation ${ }^{7}$ and procedure, we find that $\phi_{11}$ becomes $^{8}$

$$
\begin{aligned}
\phi_{11} & =\left(8 \pi^{3} b a^{1 / 2}\right)^{-1} \iiint_{-\infty}^{\infty} A_{11}\left(\omega_{1}\right) \cos \omega_{1} t \exp \left[i r_{11} \omega_{1} \cos \mu\right] \sin \mu d \mu d \nu d \omega_{1} \\
& =\left[(a-\alpha) / \pi r_{1}\right] \int_{\theta}^{\infty} C_{11}\left(\omega_{1}\right)\left\{\sin \omega_{1}\left(r_{1}-t\right)+\sin \omega_{1}\left(r_{1}+t\right)\right\} d \omega_{1} \\
& =\left(2 \pi^{2} r_{1}\right)^{-1}\left[B_{11}\left(r_{1}-t\right)+B_{11}\left(r_{1}+t\right)\right] .
\end{aligned}
$$

Thus we find that one of the possible propagation phenomena is described by a motion wherein the wave fronts are the ellipsoids $r_{1}=$ const., and whose amplitude attenuates like $1 / r_{1}$. Equation (9a) indicates that the other displacement potentials associated with $\omega_{1}$ differ from $\phi_{11}$ by a constant factor. In fact we could now write the displacements for this motion as the gradient of a single potential in a distorted coordinate system. This fact will be useful later.

The determination of the motion associated with $\omega_{2}$ requires only a slight variation on the foregcing procedure. As required by Eq. (9b), we define $A_{22}=-\zeta^{2} C\left(\omega_{2}\right) / \omega_{2}$, $A_{23}=\eta^{2} C\left(\omega_{2}\right) / \omega_{i}$, where $C\left(\omega_{2}\right)$ is derived as before from an arbitrary function $B\left(r_{2}\right)$ and $\omega_{2}$ and $r_{2}$ are defined in the same manner as were $\omega_{1}$ and $r_{1}$. The expression for $\phi_{22}$ becomes

$$
\begin{aligned}
\phi_{22} & =\frac{1}{8 \pi^{3}} \iiint_{-\infty}^{\infty} \frac{-\zeta^{2} C\left(\omega_{2}\right)}{\omega_{2}} \cos \omega_{2} t \exp [i(x \xi+y \eta+z \zeta)] d \xi d \eta d \zeta \\
& =\frac{1}{8 \pi^{2}} \frac{\partial^{2}}{\partial z^{2}} \iiint_{-\infty}^{\infty} \frac{C\left(\omega_{2}\right)}{\omega_{2}} \cos \omega_{2} t \exp [i(x \xi+y \eta+z \zeta)] d \xi d \eta_{1} d \zeta \\
& =\frac{1}{2 \pi^{2}} \frac{\partial^{2}}{\partial z^{2}}\left[\frac{B\left(r_{2}-t\right)}{r_{2}}+\frac{B\left(r_{2}+t\right)}{r_{2}}\right] .
\end{aligned}
$$

Similarly,

7 The angle $\nu$ should now be measured ahout $r_{1}$.

${ }^{8}$ The final step is due to the inverse relationship implied by Eq. (13). 


$$
\phi_{32}=-\frac{1}{2 \pi^{2}} \frac{\partial^{2}}{\partial y^{2}}\left[\frac{B\left(r_{2}-t\right)}{r_{2}}+\frac{B\left(r_{2}+t\right)}{r_{2}}\right] .
$$

The displacement $\nabla_{2}$ associated with this solution can now be written in the form

$$
\nabla_{2}=\frac{1}{2 \pi^{2}} \operatorname{curl}\left\{\mathrm{i} \frac{\partial^{2}}{\partial y \partial z}\left[\frac{B\left(r_{2}-t\right)}{r_{2}}\right]\right\} \text {. }
$$

The fact that the equations governing our problem are linear implies that the $\partial^{2} / \partial y \partial z$ of the foregoing expression is superfluous, ${ }^{9}$ that is, we may write

$$
\mathbf{v}_{2}=\frac{1}{2 \pi^{2}} \operatorname{curl}\left\{\mathrm{i} \frac{E\left(r_{2}-t\right)}{r_{2}}\right\} \text {. }
$$

The displacements corresponding to $\omega_{3}$ arise in an entirely analogous manner and are given by

$$
\mathrm{v}_{3}=\left|\begin{array}{ccc}
\mathrm{i}(b-\alpha), & \mathrm{j} \beta, & \mathrm{k} \beta \\
\frac{\partial}{\partial x}, & \frac{\partial}{\partial y}, & \frac{\partial}{\partial z}, \\
0, & \frac{P\left(r_{3}-t\right)}{r_{3}}, & \frac{Q\left(r_{3}-t\right)}{r_{3}}
\end{array}\right|,
$$

where it is required that $\partial\left(P / r_{3}\right) / \partial y+\partial\left(Q / r_{3}\right) / \partial z \equiv 0$. The first of the three foregoing solutions corresponds mathematically to the potential solutions found for the isotropic solid and the latter two to the rotational motions; together they suggest a way of factoring the equations for the displacements. We write the body forces in the following manner, choosing the coefficients of the various derivatives in the operators to correspond to those found in the foregoing results; namely

$$
(X, Y, Z)=\operatorname{grad}^{*} \Phi(x, y, z, t)+\operatorname{curl}^{*} \mathbf{M}(x, y, z, t),
$$

where

$$
\operatorname{grad}^{*}=\mathrm{i}(a-\alpha) \partial / \partial x+\mathrm{j} \beta \partial / \partial y+\mathrm{k} \beta \partial / \partial z
$$

and

$$
\operatorname{curl}^{*} \mathbf{M}=\left|\begin{array}{ccc}
\mathbf{i}(b-\alpha), & \mathbf{j} \beta, & \mathbf{k} \beta \\
\frac{\partial}{\partial x}, & \frac{\partial}{\partial y}, & \frac{\partial}{\partial z} \\
M_{x}, & M_{y}, & M_{z}
\end{array}\right| .
$$

We must require that $\partial M_{v} / \partial y+\partial M_{z} / \partial z \equiv 0$. We now assume the displacement in the form

$$
\mathbf{v}=\operatorname{grad}^{*} \phi+\operatorname{curl}^{*} \mathrm{G},
$$

substitute into equations (1), (2), and (3), and obtain a set of equations which are separable in to the following:

- Actually, all derivatives of $B / r_{2}$ constitute solutions. 


$$
\begin{gathered}
a \frac{\partial^{2} \phi}{\partial x^{2}}+b\left(\frac{\partial^{2} \phi}{\partial y^{2}}+\frac{\partial^{2} \phi}{\partial z^{2}}\right)-\frac{\partial^{2} \phi}{\partial t^{2}}=-\Phi, \\
\alpha \frac{\partial^{2} G_{x}}{\partial x^{2}}+\gamma\left(\frac{\partial^{2} G_{x}}{\partial y^{2}}+\frac{\partial^{2} G_{x}}{\partial z^{2}}\right)-\frac{\partial^{2} G_{x}}{\partial t^{2}}=-M_{x}, \\
{\left[\alpha\left(\frac{\partial^{2}}{\partial x^{2}}+\frac{\partial^{2}}{\partial y^{2}}+\frac{\partial^{2}}{\partial z^{2}}\right)-\frac{\partial^{2}}{\partial t^{2}}\right]\left(G_{y}, G_{z}\right)=-\left(M_{y}, M_{z}\right) .}
\end{gathered}
$$

The relation $\partial G_{y} / \partial y+\partial G_{z} / \partial z=0$ will be automatically satisfied.

Although the above equations may be transformed into the familiar Poisson form by trivial transformations, it is interesting to extend the foregoing procedures to obtain a formal method for the solution of, say, Eq. (23a). The steps leading to Eq. (5) transform Eq. (23a) into

$$
\left[\frac{\partial^{2}}{\partial t^{2}}+a \xi^{2}+b\left(\eta^{2}+\zeta^{2}\right)\right] \psi=T(\xi, \eta, \zeta, t),
$$

where $\psi$ is defined as before and

$$
T=\iiint_{-\infty}^{\infty} \Phi(p, q, s, t) \exp [-i(p \xi+q \eta+s \xi)] d p d q d s .
$$

Conventional operational procedures then give a particular integral of Eq. (24) as

$$
\psi=\frac{1}{\omega_{1}} \int_{0}^{t} T(\xi, \eta, \zeta, \alpha) \sin \omega_{1}(t-\alpha) d \alpha
$$

and $\phi$ becomes (according to Eq. (12)),

$$
\begin{aligned}
\phi= & \frac{1}{8 \pi^{3}} \iiint_{-\infty}^{\infty}\left[\int_{0}^{t} \Phi(p, q, s, \alpha) \iiint_{-\infty}^{\infty} \frac{\sin \omega_{1}(t-\alpha)}{\omega_{1}}\right. \\
& \cdot \exp [i\{(x-p) \xi+(y-q) \eta+(z-s) \zeta\}] d \xi d \eta d \zeta d \alpha] d p d q d s \\
= & \frac{1}{8 \pi^{3}} \iiint_{-\infty}^{\infty}\left[\int_{0}^{t} \Phi(p, q, s, \alpha) d I(\alpha)\right] d p d q d s,
\end{aligned}
$$

where $^{10}$

$$
\begin{aligned}
I(\alpha) & =\frac{1}{b a^{1 / 2}} \int_{0}^{\infty} \int_{0}^{2 \pi} \int_{0}^{\pi} \cos \omega_{1}(t-\alpha) \exp \left[i \omega_{1} \cdot R_{1}\right] \sin \mu d \mu d \nu d \omega_{1} \\
& =\frac{4 \pi}{b a^{1 / 2}} \int_{0}^{\infty} \frac{\cos \omega_{1}(t-\alpha)}{R_{1} \omega_{1}} \sin \omega_{1} R_{1} d \omega_{1} \\
& =\frac{2 \pi}{b a^{1 / 2} R_{1}} \int_{0}^{\infty} \frac{\sin \omega_{1}\left(R_{1}+t-\alpha\right)+\sin \omega_{1}\left(R_{1}-t+\alpha\right)}{\omega_{1}} d \omega_{1} .
\end{aligned}
$$

The foregoing integral is a step function which (since $0 \leqq \alpha \leqq t$, and $R_{1}>0$ ) has a single

10 Again we use the type of coordinate transformation which led to Eq. (12). 
step of magnitude $\pi$ at $\alpha=t-R_{1} . R_{1}$ is defined by $R_{1}^{2}=(x-p)^{2} / a+(y-q)^{2} / b$ $+(z-s)^{2} / b$. The evaluation of the Stieltjes integral of Eq. (26) now yields

$$
\phi=\frac{1}{4 \pi} \iiint_{-\infty}^{\infty} \frac{\Phi\left(a^{1 / 2} p, b^{1 / 2} q, b^{1 / 2} s, t-R_{1}\right)}{R_{1}} d p d q d s
$$

and we have the familiar retarded potential. The expressions for the components of $G$ can be obtained in analogous fashion.

3. More general media. It is quite evident that one might start with the general linear law relating the stresses and strains in an aerolotropic material and by the same procedures arrive at three equations analogous to Eqs. (4), using either the displacements themselves or the potentials defined in the foregoing. One would then arrive at a determinantal equation of the same form as Eq. (8). The roots could be found and Eq. (12) would be valid. However, the $\omega_{k}^{2}$ of this general problem would not appear in the concise polynomial form found in the foregoing considerations. In fact, the integrand of Eq. (12) becomes sufficiently complex in this general case that it does not seem worth while to present in more detail the procedure outlined here. 